\title{
Preface: Geometric and algebraic combinatorics
}

\author{
Edwin R. van Dam • Willem H. Haemers
}

Received: 18 April 2012 / Accepted: 14 May 2012 / Published online: 1 June 2012

(C) The Author(s) 2012. This article is published with open access at Springerlink.com

The present issue of Designs, Codes and Cryptography is devoted to the theme "Geometric and Algebraic Combinatorics". A central concept in this research area is the Association Scheme. On one hand it can be a tool for a better understanding of combinatorial objects, such as error correcting codes, block designs, point-line incidence geometries, and permutation groups. On the other hand, many association schemes are interesting objects in themselves. This includes the strongly regular and distance-regular graphs.

Algebraic tools like eigenvalues are extremely important for studying association schemes, but are also useful tools in their own right for studying the structure of graphs. Also incidence geometries, especially projective and affine geometries over finite fields are often related to association schemes, but as expected, here geometric methods play a more important role.

The issue contains fourteen articles, which we'll briefly review.

1. Simeon Ball and Jan De Beule prove part of Segre's MDS conjecture. In a large number of cases, they find an upper bound for the size of a set of vectors in $\mathbb{F}_{q}^{k}$ with the property that every subset of size $k$ is a basis. The result has implications for error correcting codes and matroid representations.

2. Dieter Jungnickel and Vladimir Tonchev generalize the Hamada type characterization of the classical point-hyperplane designs in terms of associated codes to a characterization of all classical geometric designs.

3. Sasha Jurišić and Janoš Vidali study 1-codes in distance-regular graphs with diameter 3. Using this, they prove the nonexistence of distance-regular graphs with intersection arrays in certain infinite families.

Communicated by D. Jungnickel.

E. R. van Dam $(\varangle) \cdot$ W. H. Haemers $(\varangle)$

Department of Econometrics and Operations Research, Tilburg University, Tilburg, The Netherlands e-mail: edwin.vandam@uvt.nl

W. H. Haemers

e-mail: haemers@uvt.nl 
4. Alexander Gavrilyuk and Alexander Makhnev prove the nonexistence of distance-regular graphs with intersection arrays $\{52,35,16 ; 1,4,28\}$ and $\{69,48,24 ; 1,4,46\}$, by using bounds on the size of certain substructures.

5. Jack Koolen and Jongyook Park study the relationship between the intersection number $c_{2}$ and the diameter of a distance-regular graph. They show that a distance-regular graph with large $c_{2}$ is bipartite, and that if the excess is small then the distance-regular graph is antipodal.

6. Aart Blokhuis and Andries Brouwer characterize the almost distance-regular graph on the 55 flags of the eleven point biplane by its spectrum, by examining the number of closed walks together with a study of the representation in the eigenspace for the eigenvalue -2 .

7. Aart Blokhuis, Andries Brouwer, and Willem Haemers characterize the second subconstituent of the strongly regular Brouwer-Haemers graph by its spectrum. The proof uses a strong form of eigenvalue interlacing to reduce the problem to one about root lattices.

8. Hyonju Yu determines the largest and second largest limit points of smallest eigenvalues of regular graphs less than -2 . She also determines the supremum of the smallest eigenvalue among all connected 3-regular graphs with smallest eigenvalue less than -2 and the unique graph with this supremum value as its smallest eigenvalue.

9. Hirotake Kurihara dualizes the spectral excess theorem, that is, the characterization of distance-regular graphs by its spectrum and the excess. The dual version characterizes cometric association schemes among spherical 2-designs.

10. Takuya Ikuta and Akihiro Munemasa show that the Nomura algebra of the nonsymmetric Hadamard model coincides with the Bose-Mesner algebra of the directed Hadamard graph.

11. Andries Brouwer and Çiçek Güven determine the universal embedding dimension and the generating rank of the partial linear space of short vectors in the Leech lattice and classify its hyperplanes.

12. Hendrik Van Maldeghem gives two characterizations of symplectic polarities among all dualities of buildings of type $E_{6}$. One of these completes the program for dualities of buildings of type $E_{6}$ of determining all domestic automorphisms of spherical buildings, and it also shows that symplectic polarities are the only polarities in buildings of type $E_{6}$ for which the Phan geometry is empty.

13. Bart De Bruyn determines all homogeneous pseudo-embeddings of affine and projective spaces over the field of four elements, and classifies all pseudo-hyperplanes of such affine spaces.

14. Bill Kantor and Tim Penttila characterize Desarguesian projective planes of square order by the property that every quadrangle lies on a unique Baer subplane.

The name 'Geometric and Algebraic Combinatorics', abbreviated to GAC, is also carried by a series of conferences held in Oisterwijk, The Netherlands. The papers in this special issue are related to, or inspired by talks given at GAC 5, the fifth conference in this series held from August 14 to 19, 2011. See [1-4] for papers related to GAC 1, 2, 3, and 4. The conference GAC 5, and consequently this special issue, couldn't have been established without the financial support of

- NWO, the Netherlands Organisation for Scientific Research,

- The Dutch mathematics cluster DIAMANT,

- Tilburg University (Department of Econometrics and Operations Research, and the Business and Economics Research Institute CentER). 
We are grateful to these sponsors, to all participants of the conference, and to the contributors and referees of this special issue.

Open Access This article is distributed under the terms of the Creative Commons Attribution License which permits any use, distribution, and reproduction in any medium, provided the original author(s) and the source are credited.

\section{References}

1. Des. Codes Cryptogr. 21(1-3) (2000).

2. Des. Codes Cryptogr. 34(2/3) (2005).

3. Eur. J. Comb. 28(7) (2007).

4. Eur. J. Comb. 31(6) (2010). 